\title{
Strontium ranelate in postmenopausal osteoporosis treatment: a critical appraisal
}

This article was published in the following Dove Press journal:

International Journal of Women's Health

15 December 2009

Number of times this article has been viewed

\author{
Roberto Cesareo' \\ Clemente Napolitano' \\ Mario lozzino ${ }^{2}$ \\ 'Department of Internal Medicine, \\ 2Department of Radiology, \\ S.M.Goretti Hospital, \\ Latina, Italy
}

Correspondence: Roberto Cesareo Department of Internal Medicine S.M. Goretti Hospital, Italy Tel +390773 655375 I

Fax +390773655375।

Email robertocesareo@libero.it

\begin{abstract}
Osteoporosis is a progressive and debilitating disease characterized by a massive bone loss with a deterioration of bone tissues, and a propensity for a fragility fracture. Strontium ranelate is the first antiosteoporotic treatment that has dual mode of action and simultaneously increases bone formation, while decreasing bone resorption, thus rebalancing bone turnover formation. Strontium ranelate rebalances bone turnover in favor of improved bone geometry, cortical thickness, trabecular bone morphology and intrinsic bone tissue quality, which translates into enhanced bone strength. This review describes the mechanism of the strontium ranelate action and its effects on bone mineral density, bone turnover, and osteoporotic fractures. The efficacy of strontium ranelate in postmenopausal osteoporosis treatment to reduce the risk of vertebral and hip fractures has been highlighted in several randomized, controlled trials. Treatment efficacy with strontium ranelate has been documented across a wide range of patient profiles: age, number of prevalent vertebral fractures, body mass index, and a family history of osteoporosis. Because strontium ranelate has a large spectrum of efficacy, it can be used to treat different subgroups of patients with postmenopausal osteoporosis. Strontium ranelate was shown to be relatively well tolerated and the safety aspects were good. Strontium ranelate should be considered as a first-line treatment for postmenopausal osteoporotic patients.
\end{abstract}

Keywords: osteoporosis, strontium ranelate, therapy

\section{Introduction}

Osteoporosis is a systemic skeletal disease characterized by a decreasing bone mass and a micro architectural deterioration of bone tissue, leading to a consequent increase in bone fragility and a susceptibility to fractures. ${ }^{1}$ After menopause, bone turnover acceleration induces an imbalance between bone resorption and formation, leading to a final postmenopausal bone loss. Because osteoporosis is considered to be the main cause of fractures in postmenopausal women, it represents an important and potentially preventable health problem. ${ }^{2}$

Currently available pharmacological treatments for osteoporosis modify bone micro architecture acting on one component of the bone remodelling process. Bisphosphonates reduce bone resumption, while teriparatide and other forms of parathormone act primary in the stimulation of bone formation. ${ }^{3}$

Recently, a new agent, strontium ranelate (SR), has been licensed in Europe for osteoporosis treatment. SR is the first osteoporotic treatment with a dual mode of action; it simultaneously increases bone formation and decrease bone resumption, switching the turnover equilibrium to bone formation. submit your manuscript | www.dovepress.con

Dovepress
International Journal of Women's Health 2010:2 I-6

(C) 2010 Cesareo et al, publisher and licensee Dove Medical Press Ltd.This is an Open Access article which permits unrestricted noncommercial use, provided the original work is properly cited. 
Hypothetical mechanisms of action, the effects on bone mineral density (BMD), bone turnover markers, antifracturative efficacy and safety of SR are discussed in this work.

\section{Mechanism of action}

Strontium is a bone-seeking element closely related to calcium. SR is composed of two atoms of stable strontium and one molecule of ranelic acid. ${ }^{4} \mathrm{SR}$ is a treatment for osteoporosis and unlike other drugs, has a dual effect on bone remodeling, being able to stimulate bone formation by osteoblasts, a property shared with bone-forming agents, and to inhibit bone resumption by osteoclasts, as antiresumptive agents. ${ }^{5,6}$

Despite our current knowledge on the cellular effects of SR on osteoblasts, the exact molecular mechanisms involved remain elusive. In particular the mechanism by which SR reduces fracture risk is not completely understood. It has been hypothesized that strontium could activate signaling pathways through a cation-sensing receptor which is expressed in bone cells because of its atomic and ionic properties as a divalent cation that closely resembles the calcium ion. SR could act as a calcium-like entity for the well known anabolic effects of calcium itself in bone. However preliminary data demonstrated that SR activates sensing receptors that are expressed at all stages of osteoblast activity. ${ }^{7,8}$ and there is well established evidence that strontium affects the activity of bone cells in vitro.

In vitro, SR enhances the replication of preosteoblastic cells and, secondarily, bone matrix synthesis. ${ }^{9}$ It activates gene expression in osteoblasts, and the formation of mineralized colony-forming unit-osteoblasts (CFU-obs). Bone marrow-derived stromal cells in culture, when exposed to SR, displayed a significant increase in the expression of the master gene, Runx2, as well as bone sialoprotein (BSP), and this was associated with a significant increase in the formation of CFU-obs. In particular, genes activated by SR depend on the differentiation stage: Runx2 and BSP in bone marrow-derived cells, Runx2, osteocalcin in preosteoblasts, BSP, and osteocalcin in mature osteoblasts (Figure 1). At the same time, the strontium dose dependently decreases pre-osteoclast differentiation and bone resorption. ${ }^{10,11}$ In mouse calvaria cultures, strontium inhibits dose-dependent bone resorption. ${ }^{12}$ In another study the resorbing activity of strontium was not mediated by prostaglandin $\mathrm{E}_{2}$, but osteoclast cells were required for its effect. Destruction of osteoclasts decreases strontium ${ }^{13}$ antirespective effects.

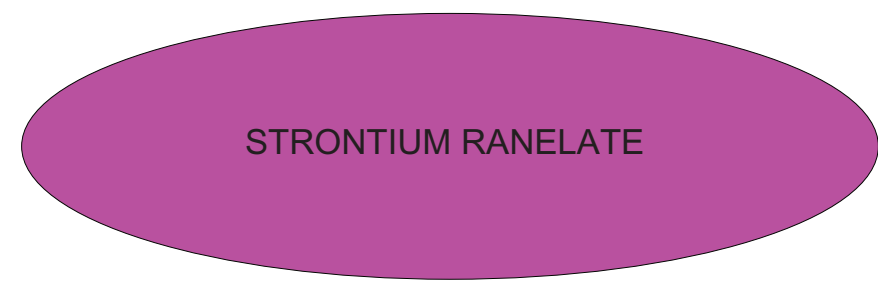

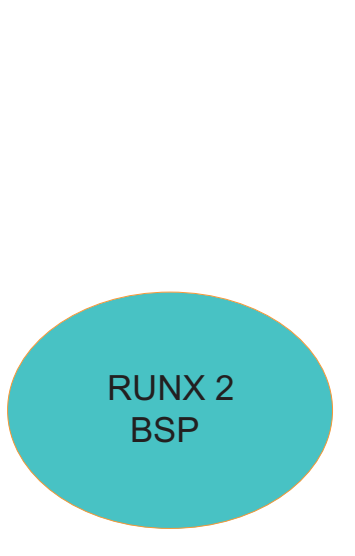

Stromal cells osteoblasts
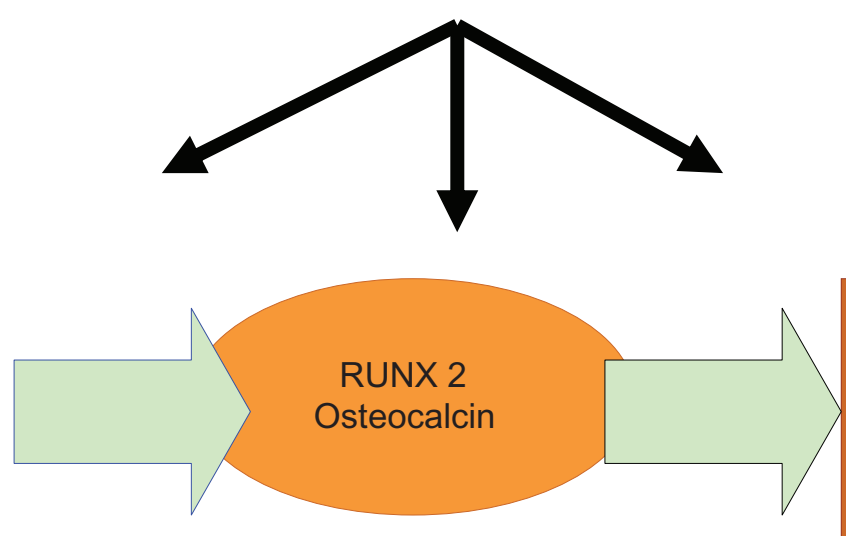

Preosteoblasts

Figure I Genes activated by strontium ranelate depend on the differentiation stage. Abbreviation: BSP, bone sialoprotein. 
In vivo studies performed in various rodent models such as immobilization-induced osteopenia and ovariectomy-induced osteoporosis in intact animals, indicate that strontium causes an imbalance of bone turnover in favor of bone formation. ${ }^{14,15}$

SR increases bone strength and this effect is related to positive influences on most of the determinants of bone strength such as bone mass, geometry, microarchitecture, and bone tissue quality. Bone static histomorphometry and microcomputed tomography demonstrated a dose-dependent increase in trabecular bone volume, trabecular number and thickness, connectivity, and cortical thickness, as assessed at the level of the tibia. SR improved bone geometry by increasing the external diameter and cortical thickness of the long bones through periosteal and endosteal apposition, respectively. Bone mechanical properties is characterized by an increase not only in the maximal load but also by a dramatic improvement in energy to failure, which was essentially due to plastic energy increase. ${ }^{16-19}$

\section{Clinical studies}

\section{Secondary endpoints: bone mineral density and bone turnover markers Bone mineral density}

In the STRATOS study, 353 osteoporotic postmenopausal women with at least one previous vertebral fracture and a lumbar T-score of less than 2.4 were randomized to receive a placebo, SR $500 \mathrm{mg}$ per day, $1 \mathrm{~g}$ per day, or $2 \mathrm{~g}$ per day. Lumbar BMD measured by dual-energy X-ray absorptiometry (DXA) was the primary parameter investigated for efficacy.

In the STRATOS study, the annual increase in lumbar BMD in the 2 g per day group $(+7.3 \%$ per year) was significantly higher than in the placebo group $(P<0.001)$. The minimum dose at which SR was effective in preventing bone loss in early postmenopausal nonosteoporotic women and in the treatment of postmenopausal osteoporosis was $1 \mathrm{~g}$ per day and $2 \mathrm{~g}$ per day, respectively. ${ }^{20}$

The SOTI trial included 1649 postmenopausal women aged 70 years on average with osteoporosis defined by a lumbar BMD of $0.731 \pm 0.125 \mathrm{~g} / \mathrm{cm}^{2}$ and/or at least one prevalent vertebral fracture (this last criterion was present in $87.5 \%$ of included patients). Over a period of three years, in the SR group a baseline of $12.7 \%$ increase in BMD was observed in the lumbar spine, $7.2 \%$ in the femoral neck, and $8.6 \%$ in the total hip $(P<0.001$ for all three comparisons with baseline values). ${ }^{21}$
The TROPOS trial included 5091 osteoporotic postmenopausal women aged 74 years (or 70 years with one risk factor for an osteoporotic fracture), with a femoral neck BMD of $<0.600 \mathrm{~g} / \mathrm{cm}^{2}$. In TROPOS, at three years in the SR group a 5.7\% baseline percentage increment in BMD was observed at the femoral neck and $7.1 \%$ at the total hip $(P=0.001)$, compared with the placebo group. ${ }^{22}$

In these clinical trials, there were impressive BMD enhancements in the spine with the spine curve similar to that for parathyroid hormone (PTH). However, some caution is necessary for a correct interpretation of these results because some of this effect could be due to the higher atomic number of strontium $(z=38)$ compared with calcium $(z=20)$ affect when BMD is measured by DXA. X-ray attenuation for strontium atoms in the bone is stronger than calcium atom attenuation. However, when the DXA scanner software calculates BMD from measured X-ray transmission factors, the increased attenuation caused by bone strontium content (BSC) is considered as calcium content attenuation and this can lead to an artificial increase in BMD. Although a commendable effort was made in the SOTI trial to correct the BMD data for the atomic number effect of strontium, there is clearly a considerable uncertainty about the accuracy of the corrections that have been done. ${ }^{21,23}$ Furthermore a strong relationship between the increase in BMD and a subsequent reduction of a new vertebral or hip fracture risk has been demonstrated in SR-treated patients, indicating that BMD level monitoring may be valuable in these patients. After treatment withdrawal, patients who switched to a placebo after four years experienced a significant reduction in BMD, showing how SR effects had been progressively reversible and reflecting the clearance of strontium from the bone. ${ }^{24}$

\section{Bone turnover markers}

In the SOTI and TROPOS studies administration of SR resulted in increased levels of serum bone alkaline phosphatase compared with the placebo group from the third month $(8.1 \% ; P=0.001)$; this difference persisted over the first three and four years of the SOTI study, while the serum telopeptide of type I collagen decreased from the third month $(<12.2 \% ; P<0.001)$. The pattern of change in these markers of bone remodeling is different from the pattern documented as occurring with antiresorptive therapies, such as bisphosphonates and selective estrogen receptor modulators (which decrease markers of bone resorption and bone formation), or with anabolic therapy such as PTH (which increases markers of bone resorption and bone formation). ${ }^{25}$ In patients treated with SR, there was a remodeling marker divergence 
(increase in bone formation, decrease in bone resorption), which underscores the difference in the mechanism of action of SR compared with traditional antiresorptive agents and paratormon. Changes were moderate, but opposite and concomitant, according with the potential mechanism of action of the drug.

\section{Primary endpoints: vertebral and nonvertebral fractures}

In the STRATOS study, there was a significant reduction of patients experiencing new vertebral deformities in the second year of treatment with $2 \mathrm{~g} / \mathrm{d}$ SR (relative risk [RR], $0.56 ; 95 \%$ confidence interval [CI]: 0.35 to 0.89 ).

In the SOTI study at the end of the first year of treatment, there was a $49 \%$ lower risk reduction of a new vertebral fracture in the SR group than in the placebo group (incidence of $6.4 \%$ versus $12.2 \%$; RR, 0.51 ; $95 \% \mathrm{CI}: 0.36$ to $0.74 ; P<0.001)$, and a $52 \%$ lower risk of symptomatic fracture (3.1\% versus. $6.4 \%$; RR, 0.48 ; $95 \%$ CI: 0.29 to 0.80 ; $P=0.003)$. Over the entire three-year study period, the SR group showed a $41 \%$ risk reduction for a new vertebral fracture than did the placebo group (20.9\% versus $32.8 \%$; RR, 0.59 ; $95 \%$ CI: 0.48 to $0.73 ; P<0.001)$. On the basis of this data, nine patients would need to be treated for three years with SR in order to prevent a vertebral fracture in one patient (95\% CI: 6 to 14). A 33\% decrease in vertebral fracture risk was observed over a four-year period (RR, 0.67; 95\% CI: 0.53 to $0.81 ; P<0.001)$.

Effective action of SR on nonvertebral fractures has been evaluated in the TROPOS trial, which included 5091 osteoporotic postmenopausal women aged 74 years (or 70 years with one risk factor of osteoporotic fracture), with a femoral neck BMD of $<0.600 \mathrm{~g} / \mathrm{cm}^{2}$.

The primary parameter of efficacy taken into consideration was the incidence of patients with at least one osteoporotic peripheral fracture. In the intention-to-treat population, SR was associated with a $16 \% \mathrm{RR}$ reduction of nonvertebral fractures over a three-year follow-up period (RR, 0.84; 95\% CI: 0.70 to $0.99 ; P=0.04)$. SR treatment was associated with a $19 \%$ risk reduction in major nonvertebral osteoporotic fractures (RR, 0.81; 95\% CI: 0.66; 0.98; $P=0.031$ ).

A $36 \%$ risk reduction of hip fracture $(\mathrm{RR}, 0.64 ; 95 \%$ CI: 0.412 to $0.997 ; P=0.046$ ) for the high-risk fracture subgroup (women $>74$ years and with femoral-neck BMD $\mathrm{T}$-score $<-3$ ) was also associated with the treatment.

Over a five-year period data came from 4935 patients for intention-to-treat analysis. There was a $15 \%$ decrease in risk for nonvertebral fractures over five years (RR, 0.85; 95\%
CI: 0.73 to $0.99 ; P=0.032$ ). There was a $43 \%$ decreased risk of hip fracture in a subset of 1128 patients with a clear indication of high risk for fractures, ie, aged 74 years or more, and a BMD t-score $\leq 2.4$ at both the lumbar spine and the femoral neck; in this subset, the risk reduction was 0.57 (95\% CI: 0.33 to $0.97 ; P=0.036) .{ }^{26}$ Although these results were obtained in a post hoc analysis, it should be pointed out that no other trial has been conducted thus far; with medication versus placebo during a five-year period, with nonvertebral fracture incidence as an end-point (Table 1).

Data from SOTI and TROPOS trials were pooled to evaluate the efficacy of SR on antivertebral fractures in women with lumbar spine (LS) osteopenia. In this case, treatment reduced the risk of vertebral fracture by $41 \%$ (RR, $0.59 ; 95 \%$ CI: 0.43 to 0.82 ), by $59 \%$ (RR, $0.41 ; 95 \%$ CI: 0.17 to 0.99 ) in the patients with no prevalent fractures, and by $38 \%$ (RR, $0.62 ; 95 \%$ CI: 0.44 to 0.88 ) in the patients with prevalent fractures. In women with osteopenia at vertebral and femoral bone, treatment reduced the risk of fracture by $52 \%$ (RR, 0.48 ; 95\% CI: 0.24 to 0.96$).{ }^{27}$

Other data from SOTI and TROPOS trials were pooled to evaluate the antifracture effective action of SR in elderly patients. In these patients ( $>80$ years) the risk of vertebral, nonvertebral, and clinical symptomatic (vertebral and nonvertebral) fractures were reduced within one year by $59 \%$ $(P=0.002), 41 \%(P=0.027)$, and $37 \%(P=0.012)$, respectively. At the end of three years, vertebral, nonvertebral, and clinical fracture risks were reduced by $32 \%(P=0.013)$, $31 \%(P=0.011)$, and $22 \%(P=0.040)$, respectively. ${ }^{28}$ The medication was well tolerated, and the safety profile was similar to that in younger patients.

Over the five years of treatment there was a relatively high drop-out (47\%) but this was similar to the drop-out

Table I Antifracture efficacy of strontium ranelate

\begin{tabular}{lll}
\hline Fracture & $\begin{array}{l}\text { Duration } \\
\text { (years) }\end{array}$ & $\begin{array}{l}\text { Risk reduction }(95 \% \\
\text { confidence interval) }\end{array}$ \\
\hline Vertebral & 1 & $0.5 I(0.36,0.74)$ \\
Vertebral & 3 & $0.59(0.48,0.73)$ \\
Vertebral & 4 & $0.67(0.53,0.8 \mathrm{I})$ \\
Clinical vertebral & $\mathrm{I}$ & $0.48(0.29,0.80)$ \\
Clinical vertebral & 3 & $0.62(0.47,0.83)$ \\
Nonvertebral & 3 & $0.84(0.70,0.99)$ \\
Nonvertebral & 5 & $0.85(0.73,0.99)$ \\
Hip (post hoc)* & 3 & $0.64(0.41,0.99)$ \\
Hip (post hoc)** & 5 & $0.57(0.33,0.97)$ \\
\hline
\end{tabular}

Notes: ${ }^{*}$ Age $>74$ years; t-score hip $\leq-2.4 ;{ }^{* *}$ Age $>74$ years; t-score hip and spine $\leq-2.4$. 
rate over three years of the clinical trial with risedronate (42\%). ${ }^{29}$ A comparison with other studies cannot be done because presently there is a lack of double blind long-term studies like this one.

\section{Safety}

$\mathrm{SR}$ is relatively well tolerated. Side effects include nausea, diarrhea, and headache, which usually resolve over time. In SOTI the most common adverse events were nausea and diarrhea (6.1\% versus $3.6 \%$ in the placebo group). The difference between the two groups disappeared after the first three months. A slight increase in the annual incidence of venous thromboembolism $(0.9 \%$ versus $0.6 \%)$ was observed at 3 years, but remained unchanged following the third year, without any underlying potential mechanism, as there is no known interaction between SR and parameters of hemostasis.

During post-marketing surveillance, isolated cases of hypersensitivity syndrome or drug rash with eosinophilia and systemic symptoms have been reported. This syndrome is associated with the appearance of skin reactions, fever and systemic findings, hypereosinophilia, hepatic abnormalities, and renal impairment. The syndrome is very rare, with severe hypersensitivity reaction occurring within 1-8 weeks from the beginning of the treatment and resulted 16/570,000 patients years exposure and two death. This can be compared with Stevens-Johnson syndrome as observed with raloxifene or bisphosphonates. ${ }^{30}$ The drug intake should be stopped if there is rash occurrence at the beginning of the treatment. This mechanism for this syndrome can not be explained. ${ }^{31,32}$

Although these are rare side effects, SR, compared with a placebo, is the only antiosteoporotic agent for which an improvement in quality of life has been detected using a sensitive osteoporosis specific questionnaire in a large prospective placebo controlled study with a 3-year follow-up period. Others antiosteoporotic agents such as alendronate and risedronate have been shown to improve quality of life although statistical relevance of the studies done is limited by the number of patients and by a short follow-up time. ${ }^{33}$

\section{Conclusion}

SR reduces vertebral, nonvertebral, and major nonvertebral fractures over 1, 3, 4, and 5 years. Its spectrum of activity covers women with osteopenia, osteoporosis, and severe osteoporosis. It is effective in reducing both vertebral and nonvertebral fractures, including hip fractures.

BMD may be used as a monitoring tool for SR, because early changes are predictive of long-term fracture reduction.
Biochemical markers of bone turnover reflect the uncoupling between resorption and formation. The safety profile in strontium ranelate is more positive if compared with other antiosteoporosis medications currently available on the market. SR should be considered as a first-line treatment for postmenopausal osteoporotic women because of its safety and effective action.

\section{Disclosures}

The authors report no conflicts of interest in this work.

\section{References}

1. Consensus development conference: diagnosis, prophylaxis, and treatment of osteoporosis. Am J Med. 2003;94:646-650.

2. Dennison E, Cooper C. Epidemiology of osteoporotic fractures. Horm Res. 2000;54:58-63.

3. Cesareo R, Iozzino M, Alva D, et al. Evidence based medicine and effective interventions of pharmacological therapy for the prevention of osteoporotic fractures. Min Endocrinol. 2007;32:275-295.

4. Marie P. Strontium ranelate: a novel mode of action optimizing bone formation and resorption. Osteoporos Int. 2005;16:7-10.

5. Marie P. Strontium ranelate: a dual mode of action rebalancing bone turnover in favor of bone formation. Curr Opin Rheumatol. 2006;S18:11-15.

6. Bonnelye E, Chabadel A, Saltel F, et al. Dual effect of strontium ranelate: stimulation of osteoblast differentiation and inhibition of osteoclast formation and resorption in vitro. Bone. 2008;42:129-138.

7. Coulombe J, Faure H, Robin B, et al. In vitro effects of strontium ranelate on the extracellular calcium-sensing receptor. Biochem Biophys Res Commun. 2004;323:1184-1190.

8. Chattopadhyay N, Quinn SJ, Kifor O, et al. The calcium-sensing receptor $(\mathrm{CaR})$ is involved in strontium ranelate-induced osteoblast proliferation. Biochem Pharmacol. 2007;74:438-447.

9. Canalis E, Hott M, Deloffre P, et al. The divalent strontium salt S12911 enhances bone cell replication and bone formation in vitro. Bone. 1996; 18:517-523.

10. Marie P. Strontium ranelate: a dual mode of action rebalancing bone turnover in favor of bone formation. Curr Opin Rheumatol. 2006;18: S11-S15.

11. Zhu LL, Zaidi S, Peng Y, et al. Induction of a program gene expression during osteoblast differentiation with strontium ranelate. Biochem Biophys Res Commun. 2007;355:307-311.

12. Marie PJ, Hott M, Modrowski D, et al. An uncoupling agent containing strontium prevents bone loss by depressing bone resorption and maintaining bone formation in estrogen-deficient rats. $J$ Bone Miner Res. 1993;8:607-615.

13. Zaidi M, Adebanjo OA, Moonga BS, et al. Emerging insights into the role of calcium ions in osteoclast regulation. $J$ Bone Miner Res. 1999; 14:669-674.

14. Marie PJ, Hott M, Modrowski D, et al. An uncoupling agent containing strontium prevents bone loss by depressing bone resorption and maintaining bone formation in estrogen-deficient rats. $J$ Bone Miner Res. 1993;8:607-615.

15. Delannoy P, Bazot D, Marie PJ. Long-term treatment with strontium ranelate increases vertebral bone mass without deleterious effect in mice. Metabolism. 2002;51:906-911.

16. Ammann P, Barraud S, Dayer R, et al. Strontium ranelate treatment improves trabecular and cortical intrinsic bone tissue quality, a determinant of bone strength. J Bone Miner Res. 2007;22:1419-1125.

17. Ammann $P$, Shen V, Robin B, et al. Strontium ranelate improves bone resistance by increasing bone mass and improving architecture in intact female rats. J Bone Miner Res. 2004;19:2012-2020. 
18. Arlot ME, Jiang Y, Genant HK, et al. Histomorphometric and $\mu \mathrm{CT}$ analysis of bone biopsies from postmenopausal osteoporotic women treated with strontium ranelate. J Bone Miner Res. 2008;23:215-222.

19. Ammann P, Badoud B, Barraud S, et al. Strontium ranelate treatment improves trabecular and cortical intrinsic bone tissue quality, a determinant of bone strength. J Bone Miner Res. 2007;22:1419-1425.

20. Meunier PJ, Slosman DO, Delmas PD, et al. Strontium ranelate: dosedependent effects in established postmenopausal vertebral osteoporosis a 2-year randomized placebo controlled trial. J Clin Endocrinol Metab. 2002;87:2060-2066.

21. Meunier PJ, Roux C, Seeman E, et al. The effects of strontium ranelate on the risk of vertebral fracture in women with postmenopausal osteoporosis. $N$ Engl J Med. 2004;350:459-468.

22. Reginster JY, Seeman E, De Vernejoul MC, et al. Strontium ranelate reduces the risk of nonvertebral fractures in postmenopausal women with osteoporosis: Treatment of Peripheral Osteoporosis (TROPOS) study. J Clin Endocrinol Metab. 2005;90:2816-2822.

23. Nielsen SP, Slosman D, Sorensen OH, et al. Influence of strontium on bone mineral density and bone mineral content measurements by dual X-ray absorptiometry. J Clin Densitom. 1999;2:371-379.

24. Bruyere O, Roux C, Detilleux J, et al. Relationship between bone mineral density changes and fracture risk reduction in patients treated with strontium ranelate. J Clin Endocrinol Metab. 2007;92:3076-3081.

25. Heaney RP. Advances in therapy for osteoporosis. Clin Med Res. 2003;1:93-99.
26. Reginster JY, Felsenberg D, Boonen S, et al. Effect of long-term strontium ranelate treatment on the risk of nonvertebral and vertebral fractures in postmenopausal women osteoporosis. Arthritis Rheum. 2008;58:1687-1695.

27. Seeman E, Vellas B, Benhamou C, et al. Strontium ranelate reduces the risk of vertebral and non vertebral fractures in women eighty years of age and older. J Bone Miner Res. 2006;21:1113-1120.

28. Seeman E, Devogelaer JP, Lorenc R, et al. Strontium ranelate reduces the risk of vertebral fractures in patients with osteopenia. J Bone Miner Res. 2008;23:433-438.

29. Sorensen OH, Crawford GM, Mulder DJ, et al. Long-term efficacy of risedronate: a 5 year placebo-controlled clinical experience. Bone. 2003;32:120-126.

30. Cole Z, Dennison E, Cooper C. Update on the treatment of post-menopausal osteoporosis. Br Med Bull. 2008;86:1-15.

31. Pernicova I, Middleton ET, Aye M. Rash, strontium ranelate and DRESS syndrome put into perspective. European Medicine Agency on the alert. European Medicine agency on the alert. Osteoporos Int. 2008;19:1811-1812.

32. Cole Z, Dennison E, Cooper C. Update on the treatment of post-menopausal osteoporosis. Br Med Bull. 2008;86:129-143.

33. Marquis P, Roux C, de la Loge C, et al. Strontium ranelate prevents quality of life impairment in post-menopausal women with established vertebral osteoporosis. Osteop Int. 2008;19:503-510.
International Journal of Women's Health

\section{Publish your work in this journal}

The International Journal of Women's Health is an international, peerreviewed open-access journal publishing original research, reports, reviews and commentaries on all aspects of women's healthcare including gynaecology, obstetrics, and breast cancer. Subject areas include: Chronic conditions (migraine headaches, arthritis, osteoporosis);

\section{Dovepress}

Endocrine and autoimmune syndromes; Sexual and reproductive health; Psychological and psychosocial conditions. The manuscript management system is completely online and includes a very quick and fair peer-review system. Visit http://www.dovepress.com/ testimonials.php to read real quotes from published authors. 